\title{
Diffusion Weighted Imaging
}

National Cancer Institute

\section{Source}

National Cancer Institute. Diffusion Weighted Imaging. NCI Thesaurus. Code C111116.

A diffusion MRI technique in which diffusion-sensitizing gradients are applied to the imaging sequence. 